山्山FANÇAISE

$>\mathrm{DE}$

$\stackrel{1=1}{\simeq}$ PÉDAGOGIE

\section{Revue française de pédagogie}

Recherches en éducation

162 | janvier-mars 2008

Acquisitions et progressions scolaires : recherches en psychologie

\title{
RousSEAU Patrick. Pratique des écrits et écriture des pratiques. La part « indicible » du métier d'éducateur
}

Paris : L'Harmattan, 2007. - 296 p.

\section{Gilles Monceau}

\section{(2) OpenEdition \\ Journals}

\section{Édition électronique}

URL : http://journals.openedition.org/rfp/972

DOI : $10.4000 /$ rfp. 972

ISSN : 2105-2913

Éditeur

ENS Éditions

\section{Édition imprimée}

Date de publication : 1 janvier 2008

Pagination : 149-150

ISBN : 978-2-7342-1118-1

ISSN : 0556-7807

\section{Référence électronique}

Gilles Monceau, «Rousseau Patrick. Pratique des écrits et écriture des pratiques. La part « indicible » du métier d'éducateur », Revue française de pédagogie [En ligne], 162 I janvier-mars 2008, mis en ligne le 29 septembre 2010, consulté le 25 septembre 2020. URL : http://journals.openedition.org/rfp/972 ; DOI : https://doi.org/10.4000/rfp.972

Ce document a été généré automatiquement le 25 septembre 2020.

(c) tous droits réservés 


\title{
ROUSSEAU Patrick. Pratique des écrits et écriture des pratiques. La part « indicible » du métier d'éducateur
}

Paris : L'Harmattan, 2007. - 296 p.

\author{
Gilles Monceau
}

\section{RÉFÉRENCE}

RousSEAU Patrick. Pratique des écrits et écriture des pratiques. La part « indicible » du métier d'éducateur. Paris : L'Harmattan, 2007. - 296 p.

1 Cet ouvrage résulte d'une thèse en sciences de l'éducation soutenue en 2006 sous la direction de Michel Corbillon. Il s'agit d'une recherche menée dans deux services de l'action éducative en milieu ouvert (AEMO) avec la participation active de vingt éducateurs en charge de suivis éducatifs prononcés par des juges pour enfants. L'auteur est lui-même un professionnel du secteur dans lequel il exerce des fonctions d'encadrement. Comme il l'écrit (p. 91) sa posture de recherche est celle d'un " praticien chercheur » qui mène de front une carrière professionnelle et des travaux de recherche supports de diverses publications. Signalons d'ailleurs sa contribution en 2003 à un ouvrage qui portait déjà sur l'écriture des pratiques professionnelles ${ }^{1}$ et dans lequel il faisait part de ses premières observations sur les paradoxes de l'écriture des rapports rédigés par les éducateurs de l'AEMO.

2 Contrairement à ce que le titre de l'ouvrage pourrait laisser entendre, l'objectif de la recherche est davantage de développer une meilleure connaissance des pratiques professionnelles des éducateurs, dont la production de notes et de rapports, que d'interroger spécifiquement la part que tient l'écriture dans ces pratiques. Le double intérêt de l'ouvrage consiste donc en la construction d'un dispositif de recherche exigeant qui use de l'écriture comme outil de collecte des données et dans ce qu'il 
montre des aspects paradoxaux du travail éducatif accompli dans les services en question.

3 Les deux premiers chapitres consistent en un repérage théorique qui définit un cadre de référence et quelques présupposés de la recherche entreprise. La problématique de l'écriture au travail est articulée à celle de l'identité professionnelle pour mettre en évidence l'intérêt du concept de paradoxe, référé à Y. Barel, pour explorer l'indicible des pratiques d'AEMO. C'est ensuite le questionnement ancien des rapports entre travail social et contrôle social qui est revisité pour en interroger la pertinence dans le cas des services sociaux concernés dont l'activité peut être décrite comme combinant évaluation des situations, contrôle et régulation.

4 La seconde partie de l'ouvrage présente le terrain de l'AEMO, ses fondements et ses cadres administratif et judiciaire actuels. Cette présentation du contexte institutionnel permet de mieux cerner la pertinence du concept de paradoxe, plutôt que celui de contradiction, pour penser les pratiques étudiées. C'est ensuite le dispositif de recherche lui-même qui est présenté, dispositif d'un grand intérêt dont les principes pourraient utilement inspirer bien des praticiens qui se font chercheurs dans leur propre champ professionnel. L'écriture y tient un double statut car le corpus est constitué de deux ensembles distincts et complémentaires. D'une part le chercheur a convaincu une vingtaine d'éducateurs de rédiger des livrets de suivi des mesures éducatives qui leur étaient confiées, trente et un seront réalisés sur une moyenne de neuf mois et demi chacun. D'autre part, cinquante cinq notes et rapports rédigés à l'intention des partenaires institutionnels du service, en particulier les juges pour enfants, dans le cadre de ces suivis complètent le corpus. Les deux ensembles sont donc bien différents : des écrits produits à la demande du chercheur et des écrits produits dans le cadre de la pratique ordinaire.

5 Pour rendre compte des pratiques à travers l'analyse du corpus, trois «catégories thématiques » ont été définies : observation, intervention et réflexion. Les exemples donnés permettent au lecteur de se familiariser avec ces trois catégories tout en lui permettant d'approcher de plus près la complexité des actes de ces professionnels.

6 La troisième partie de l'ouvrage est constituée de l'analyse du corpus. En montrant de quelle manière il traite les écrits produits par les professionnels, le chercheur met en évidence le fait que les éducateurs se trouvent fréquemment en situation de devoir s'appuyer sur des observations, directes ou qui leur ont été rapportées, pour soutenir leurs propres interventions auprès des familles. C'est alors tout l'indicible, et l'indécidable, de l'exercice quotidien de l'éducation en milieu ouvert qui apparaît de manière détaillée, à travers les divers exemples donnés. Les analyses ne tombent ni dans le misérabilisme ni dans le discours idéologique. Les matériaux collectés sont examinés avec précision mais sans froideur. Disons le, le style de l'auteur lui-même est son premier atout pour rendre compte de ses résultats. Son propre travail d'écriture semble avoir tiré profit à la fois de l'expérience du travailleur social, qui doit faire avec des situations difficiles tout en conservant par rapport à elles une distance permettant de les penser, et de la formation universitaire qui lui a permis de construire une posture d'objectivation des pratiques par lesquelles ses collègues travailleurs sociaux objectivent les situations familiales dans lesquelles ils interviennent et qu'ils évaluent.

7 L'auteur a su intégrer l'analyse critique du travail social dans sa manière d'en rendre compte. Ainsi, il évite les aveuglements que pourraient générer ses implications professionnelles sans pour autant tenir un discours à charge sur des pratiques dont il 
montre à la fois les fragilités et les risques. C'est en particulier ce qu'il fait à partir des travaux concernant la dimension de contrôle du travail social, ce qu'il prolonge par la question ardue de l'évaluation des situations familiales par des travailleurs sociaux qui ont à émettre des diagnostics et des pronostics. Là encore, c'est le choix de montrer la complexité et le paradoxe qui prend le pas sur la formulation de conclusions globalisantes.

8 Le livre de P. Rousseau fait pénétrer le lecteur dans l'intimité des pratiques plus qu'il n'interroge le processus de professionnalisation du métier lui-même ${ }^{2}$. On comprend aussi, à la lecture, qu'il soit si difficile aux professionnels de rendre compte de leur activité tant leurs perceptions des enjeux personnels et familiaux d'autrui sont sans cesse engagées. C'est donc à une plongée dans la production quotidienne d'actes professionnels complexes que nous sommes invités. En écartant le recours aux énoncés oraux des professionnels pour privilégier les traces écrites qu'ils laissent de leurs actes, le dispositif de recherche tranche avec une orientation dominante de l'analyse des pratiques professionnelles qui fait de la parole le vecteur privilégié de l'analyse.

Bien que l'on puisse regretter que ces analyses des productions écrites n'aient pas été restituées aux professionnels dans le cadre de la recherche, on ne peut que constater la richesse de l'éclairage apporté par ce travail qui vient compléter par sa précision factuelle bien des analyses qui s'en tiennent aux déclarations des individus. Le travail de P. Rousseau nous invite donc à prendre en compte les écrits professionnels dans l'analyse des pratiques mais surtout à construire des dispositifs de recherche dans lesquels l'écriture soit suscitée. Il contribue ainsi, avec d'autres ${ }^{3}$, à une utile diversification des démarches de recherche.

Le praticien chercheur peut donc tirer profit de ses implications institutionnelles pour élaborer un dispositif exigeant qui s'inspire des démarches de la recherche-action. Les résultats qu'il produit ainsi s'offrent comme matériaux réflexifs pour les professionnels et comme une source précieuse de données et de cadres d'analyse pour les chercheurs.

\section{NOTES}

1. CoRbillon M. (2003). « Les stratégies langagières du rapport d'AEMO » in C. Blanchard Laville \& D. Fablet. Écrire les pratiques professionnelles. Paris : L'Harmattan.

2. CRINON J. \& GUIGUE M. (2006). «Écriture et professionnalisation », Revue française de pédagogie, $\mathrm{n}^{\circ} 156$.

3. C IfALI M. \& A NDRE A. (2007). Écrire l'expérience. Vers la reconnaissance des pratiques professionnelles. Paris : PUF. 


\section{AUTEURS}

\section{GILLES MONCEAU}

ESSI, université Paris 8 